\title{
The Mass Analyzer for Real-time Investigation of Neutrals at Europa (MARINE)
}

\author{
Murray Darrach \\ Jet Propulsion Laboratory, \\ California Institute of Technology \\ 4800 Oak Grove Dr. \\ Pasadena, CA 91109 \\ 818-393-2689 \\ Murray.R.Darrach@jpl.nasa.gov
}

Andres Jaramillo-Botero

California Institute of Technology

1200 E. California Blvd.

Pasadena, CA 91125

626- 395-3591

ajaramil@caltech.edu

\author{
Stojan Madzunkov, Rembrandt \\ Schaefer Dragan Nikolic, \\ Jurij Simcic, Richard Kidd, \\ Evan Neidholdt \\ Jet Propulsion Laboratory, \\ California Institute of \\ Technology
}

\author{
Marcin Pilinski \\ ASTRA LLC \\ 5777 Central Ave. Ste 221 \\ Boulder, CO 80301 \\ 303-720-1573 \\ mpilinski@astraspace.net
}

\author{
Ken Farley \\ California Institute of Technology \\ 1200 E. California Blvd \\ Pasadena, CA 91125 \\ 626-395-6055 \\ farlev@gps.caltech.edu
}

\begin{abstract}
Presented herein is the progress on developing a new mass analyzer for analysis of the exospheres of planets, moons, and primitive bodies, such as found at Europa or Enceladus. Europa, one of Jupiter's four Galilean moons, may have a subsurface ocean plausibly containing the key ingredients for life as well as sources of chemical energy. Clues to the composition and chemical state of Europa's ocean can be found both on Europa's icy surface and in its tenuous atmosphere. Given the high scientific priority of assessing the habitability of Europa's ocean, the Europa Clipper notional payload includes a Neutral Mass Spectrometer whose purpose is to characterize the composition of ejected surface products during a series of flyby investigations.
\end{abstract}

The Mass Analyzer for Real-time Investigation of Neutrals at Europa (MARINE) is capable of measuring the abundances of neutral particle species in Europa's exosphere including $\mathrm{H}_{2} \mathrm{O}, \mathrm{O}_{2}, \mathrm{CO}_{2}$, and $\mathrm{SO}_{2}$, and determining their number density profiles at per-second sampling rates as a function of altitude above Europa's surface. MARINE will either detect tracers of potential subsurface biological activity in Europa's exosphere, or place upper limits on their surface abundances. It exceeds all requirements for the proposed investigations with margins ranging from 100 to $1000 \%$, while remaining fully compatible with spacecraft accommodation constraints for mass, power, data volume, and field-of-view.

\section{TABLE OF CONTENTS}

1. INTRODUCTION .1

2. OBJECTIVES AND REQUIREMENTS ....................1

3. MARINE DESIGN.........................................3

4. Performance Testing ................................6

5. Modeled Performance at Europa .............7

6. SUMMARY .......................................................99

7. REFERENCES....................................................9

8. BIOGRAPHY ...................................................9

\section{INTRODUCTION}

The composition and spatial structure of Europa's exosphere contains critical data bearing on the moon's 978-1-4799-5380-6/15/\$31.00 C2015 IEEE history, surface processes, and possibly on the composition and habitability of its subsurface ocean. However, making compositional measurements of the exosphere is challenging: the exosphere is extremely tenuous, hypervelocity flybys leave little time to acquire signal, the list of exospheric species is largely unknown but potentially diverse, and measurements will undoubtedly be partially obscured by spacecraft $(\mathrm{S} / \mathrm{C})$ offgassing and radiation-induced noise. Clearly, any instrument designed to characterize Europa's exospheric composition must have extraordinary capabilities.

The Mass Analyzer for Real-time Investigation of Neutrals at Europa (MARINE) instrument offers scientifically compelling in situ chemical characterization of the Europan exosphere. Using the multiple flyby encounters, MARINE will develop a 3D compositional map of neutral volatiles in the exosphere that will provide a wealth of information to inform diverse scientific questions. Since so little is currently known about the exosphere, and more importantly about Europa's surface from which it derives, MARINE is designed to maximize the potential for discovery. MARINE accomplishes this exploration objective without compromising the ability to test and refine specific hypotheses on the origin of the exosphere and its relationship to the surface and possibly the deep interior, and on the history and origin of the moon. A typical operational profile for MARINE during an Europan encounter is shown in Figure 1.

\section{OBJECTIVES AND REQUIREMENTS}

MARINE's mapping of the exosphere addresses key science goals in the Europa mission Science Traceability Matrix (STM) [1] and the 2011 Planetary Decadal Survey, Vision and Voyages [2]. MARINE measurements are complementary to the many types of measurements (e.g. radar and thermal surface observations) likely to be obtained on a Europa mission. MARINE would also complement other in situ exosphere measurements such as impact analysis of ice particles in potential plumes. There are two main 
objectives to the MARINE investigation at Europa, which are:

Objective \#1: To document the major and trace constituents in Europa's ice shell via volatiles delivered to the exosphere to assess the habitability of Europa's ocean.

Characterization of the chemical composition of Europa's surface, subsurface and ocean is a critical science objective for any Europa mission. An accounting of volatiles derived from the ice shell will provide key insight to the origin and reaction pathways of Europa's surface. This objective specifically seeks to assess whether any exospheric components are present that appear to derive from the subsurface ocean, and if so, whether their composition is consistent with a habitable environment or even might contain evidence of extant life. MARINE will look for the coexistence of redox couples from which energy for metabolism can be derived (e.g. $\mathrm{H}_{2} \mathrm{~S}-\mathrm{SO}_{2}$ or $\mathrm{CH}_{4}-\mathrm{CO}_{2}$ ), as well as volatile organic molecules that are plausible building blocks or indicators of life (e.g. alkanes, carboxylic acids, alcohols). The analysis of such species in the exosphere must begin with a detailed exploration and characterization of what is present, coupled with development and validation of appropriate exospheric production and surface modification models. If any such species are indeed detected, then this

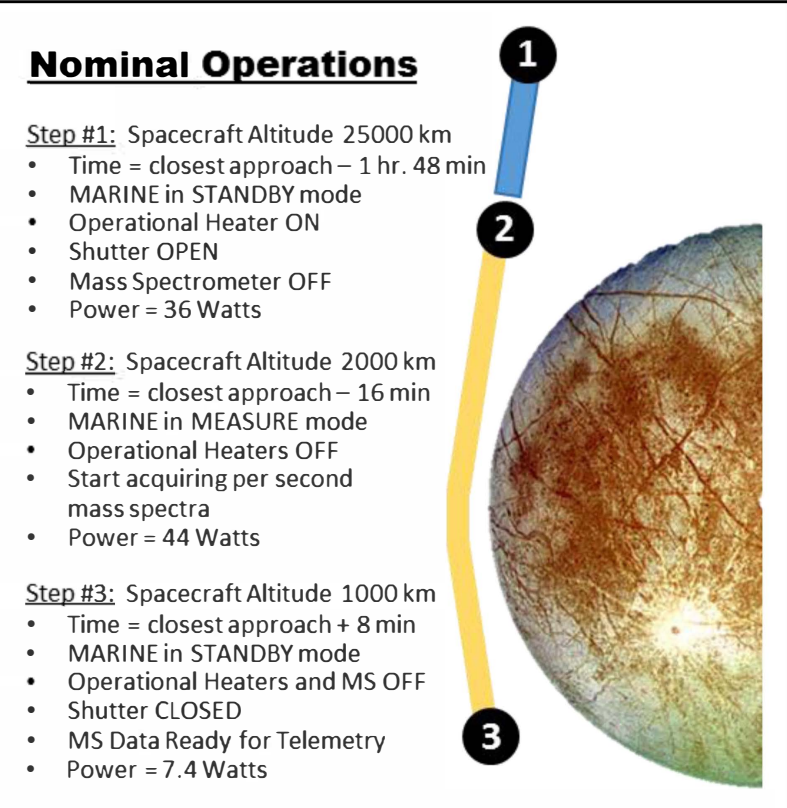

Figure 1. Example of a MARINE Operations Plan for a Europa Flyby. For the type of encounter detailed in the figure, MARINE's power consumption is 83.7 W•hr. MARINE investigations include, abundance profile measurements of the major exospheric species, plume detection and characterization, correlation of exospheric constituents to surface features, and measurements of key isotopic ratios (e.g. ${ }^{16} \mathrm{O}:{ }^{17} \mathrm{O}$ ) to understand Europa's planetary evolution.
MARINE data will provide the first step for carefully investigating their origin.

This objective requires the broadest possible range of volatile species with the best possible detection limit. MARINE proposes a science detection limit equivalent to $10^{-5}$ times that of the model exospheric water abundance, roughly corresponding to an abundance of $10 \mathrm{ppm}$ in the ice surface. The mass range of interest encompasses everything from the ${ }^{12} \mathrm{C}$ fragment up through $\sim 300 \mathrm{Da}$. This encompasses a variety of possible organic molecules, including long chain organics ( $\sim 14$ to $\sim 20 \mathrm{C}$ atoms). The investigation also requires a mass resolution of no less than $\mathrm{m} / \Delta \mathrm{m}=300$ at $300 \mathrm{Da}$. Higher detection sensitivities for trace species can be obtained by integrating signal along the entire path of a flyby.

Objective \#2: To detect and characterize localized phenomena (e.g. possible plumes) and correlate detected exospheric species to geomorphic features (e.g. fractures)

Possible Europan plumes represent a very difficult problem for unambiguous detection and characterization of their constituents. Due to their limited geometric extent and possible ephemeral nature, the utmost real-time detection sensitivity is required. It is also required that the MS sample inlet and sensor systems be specifically designed to respond quickly to changes in $\mathrm{H}_{2} \mathrm{O}$ abundance along the spacecraft trajectory.

Observations of Europa have suggested plumes extending to at least $200 \mathrm{~km}$ altitude and $200 \mathrm{~km}$ in lateral extent [3]. As such, it is required that the MS have a measurement cadence to provide a "ground-track" resolution of $\sim 25 \mathrm{~km}$, which would give multiple measurements of density and composition across a $200 \mathrm{~km}$ extent. Other than water vapor, nothing is known of the composition of a possible Europan plume. Thus, assuming Enceladus's plumes [4] as an analog for the targets of interest, the species list would include inorganic (e.g. $\mathrm{NH}_{3}, \mathrm{H}_{2} \mathrm{~S}, \mathrm{~N}_{2}, \mathrm{CO}$ ) and organic molecules (e.g. $\mathrm{CH}_{4}, \mathrm{CH}_{3} \mathrm{OH}$, and other hydrocarbons). The MARINE high resolution (HR) mode produces mass spectra from 10 to $80 \mathrm{Da}$ with the mass resolution greater than that required to resolve key species (e.g. ${ }^{32} \mathrm{~S}$ and ${ }^{16} \mathrm{O}^{16} \mathrm{O}(\mathrm{m} / \Delta \mathrm{m}$ at $50 \%$ max. peak height $\approx 1800), \mathrm{H}_{2}{ }^{32} \mathrm{~S}$ and ${ }^{34} \mathrm{~S}(\mathrm{~m} / \Delta \mathrm{m}$ at $50 \%$ max. peak height $\approx 1700), \mathrm{H}_{2}{ }^{32} \mathrm{~S}$ and ${ }^{16} \mathrm{O}^{18} \mathrm{O}(\mathrm{m} / \Delta \mathrm{m}$ at $50 \%$ max. peak height $\approx 2000$ )).

It is clear that understanding the nature and formation mechanisms of the many surface features on Europa, including possible sites of recent or current activity, are critical science objectives [2]. There is persuasive evidence that Europa's exosphere is directly coupled to the underlying surface via the sputtering process. As a result, the composition of the exospheric column above a particular surface location or feature likely carries key information about its chemical properties. By correlating MARINE's high-resolution 3D compositional maps of the exosphere to imaging data (e.g. radar, topographic, thermal), MARINE may offer the key to deciphering the relative contributions of 
various sources to Europa's surface and to the origin of the features themselves. It may also offer the ability to assess, understand, and model the effects of surface processing (e.g. sorption, sputtering, radiolysis, and impact gardening) [5, 6]. MARINE has the capability to make high-sensitivity, spatially-resolved chemical measurements - precisely what is required for this exploratory work. MARINE could potentially identify surface-exosphere features from 30 to $300 \mathrm{~km}$ in size. MARINE satisfies all of its exospheric constituent and altitude profiling science objectives following at least a single flyby encounter with a closest approach of $25 \mathrm{~km}$. As potential Europan plumes or surfaceexosphere phenomenon may be transitory or geographically constrained, MARINE will employ all spacecraft encounters at, or below, $100 \mathrm{~km}$ altitudes to satisfy these type of science objectives.

\section{MARINE DESIGN}

MARINE has a simple design whose performance has been matured and validated through construction and testing of a prototype (PT) unit, specifically for the Europa mission under a 2013 Instrument Concepts for Europa Exploration (ICEE) award. A simplified block diagram of the MARINE instrument is shown in Figure 2 and a computer aided design (CAD) model of the MARINE Sensor Head Assembly (MSHA) shown in Figure 3. Photographs of the PT MARINE MSHA and MARINE Sensor Electronics Assembly are shown in Figure 4.

During a nominal Europa flyby, the MARINE instrument will collect and concentrate through ram enhancement, the exospheric species by using a simple passive neutral collection (PNC) funnel. Ions below $300 \mathrm{eV}$ in energy are repelled by grids and do not enter the PNC funnel. The neutral species then enter the quadrupole ion trap mass spectrometer (QITMS) where they are ionized by an electron beam, and trapped in an $r f$ potential field. These ions are then mass analyzed by being selectively ejected from the QITMS and detected by a charged particle detector. The passive neutral collection (PNC) funnel is protected from dust and micro-meteor impacts during the long cruise phase through use of a shutter with a "fail-open" architecture.

Under ICEE funding, the PNC was designed by JPL and ASTRA (Atmospheric \& Space Technology Research Associates) Corp. to achieve the concentration enrichment desired for analysis of Europa's tenuous exosphere and the fast response necessary to map those signals to sources on the surface. The grazing incidence funnel (inlet diameter $=5.2$ $\mathrm{cm}$, half angle $=10^{\circ}$ ) has an interior surface coated with a 5 $\mu \mathrm{m}$ Au layer to mitigate hypervelocity impact chemistry. The PNC has been optimized for conductance and transmission time using a numerical test-particle code, the Statistical Program for Aerodynamic and Radiation Coefficient Simulation (SPARCS) [7]. Conductance (Ca) is defined as the ratio of the simulated total flux through the exit aperture of the PNC to the effusive flux though a simple aperture of the same dimension as the PNC exit aperture, when exposed to the same free-stream properties. SPARCS modeling was

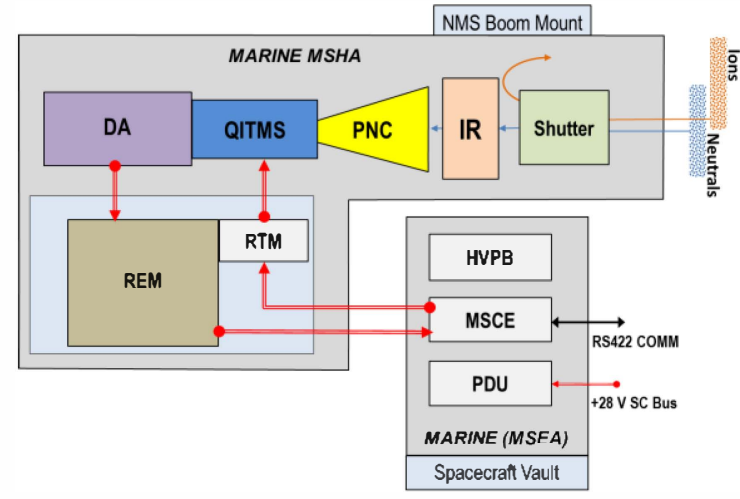

Figure 2. A Simplified Block Diagram of the MARINE Instrument. The three "6U form factor" electronics cards: Power Distribution Unit (PDU), Mass Spectrometer Control Electronics (MSCE), and High Voltage Power Board (HVPB) comprise the MARINE Sensor Electronics Assembly (MSEA). The MSEA is accommodated in the spacecraft electronics vault. The MARINE Sensor Head Assembly (MSHA) is accommodated on the Neutral Mass Spectrometer boom. The MSHA is comprised of the: Remote Electronics Module (REM), Remote Tank Module (RTM), Detector Assembly (DA), Quadrupole Ion Trap Mass Spectrometer (QITMS), Passive Neutral Collector (PNC), Ion repeller (IR), and the shutter.

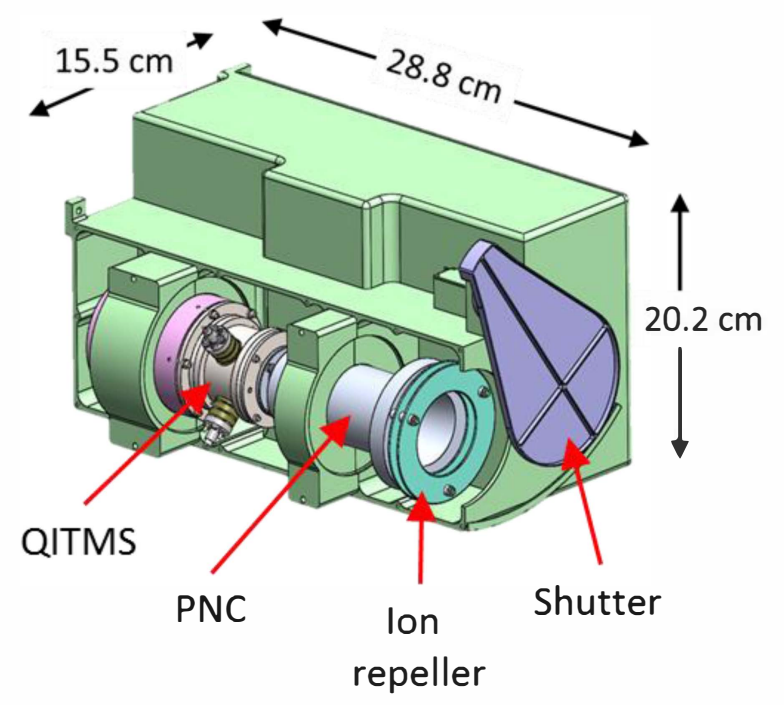

Figure 3. CAD Model of the MARINE MSHA. The MSHA uses a Passive Neutral Collector (PNC) to funnel neutrals into an ultra-sensitive Quadrupole Ion Trap Mass Spectrometer (QITMS). The PNC inner surfaces are protected during the long cruise phase by an actuated paddle shutter. The Current Best Estimate (CBE) mass of the MSHA $=5.9 \mathrm{~kg}$. 

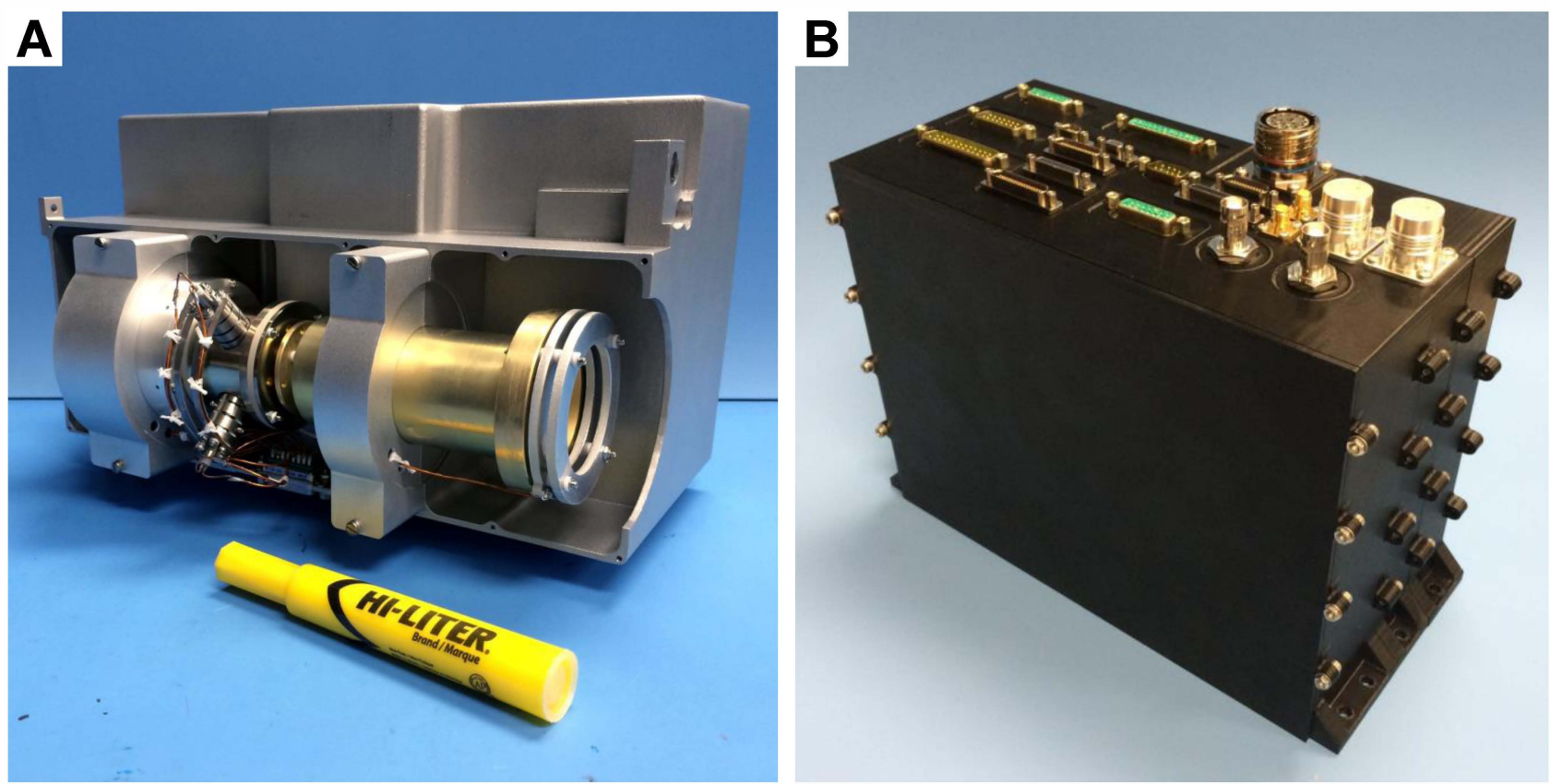

Figure 4. Photograph of the prototype (PT) MARINE subassemblies. (A) MARINE Sensor Head Assembly (MSHA). The MSHA cover is removed for clarity. (B) MARINE Sensor Electronics Assembly (MSEA). The MARINE MSHA is accommodated on the Europa spacecraft Neutral Mass Spectrometer (NMS) boom mount and the MARINE MSEA inside the spacecraft vault. The PT prototype sensor and electronics were developed and tested under the 2013 ICEE award. CBE mass of the MSHA and MSEA are $5.9 \mathrm{~kg}$ and $3.0 \mathrm{~kg}$, respectively. Testing of the MARINE PT unit has verified the design for all performance and accommodation specifications

performed to ensure robustness against the wide variety of scattering phenomena expected during Europa flybys, including concentration as a function of angle-of-attack $\left(0^{\circ}-\right.$ $90^{\circ}$ ), molecular vertical velocities and temperature, concentrator half-angle, and diffuse/quasi-specular/specular scattering models. A worst-case ram enhancement of $\mathrm{Ca}=30$ was found for species chemically similar to atomic oxygen (highly reactive) yielding diffuse-like reflections. Best-case ram enhancements of $\mathrm{Ca}=120$ were found for specular reflections by neutral gas species (e.g. $\mathrm{CO}_{2}$ ). The modeled ram enhanced PNC density and resultant QITMS partial pressure as a function of altitude (and thus angle) is shown in Fig. 5, assuming worst-case collision dynamics $(\mathrm{Ca}=30)$. However, under the free-stream conditions and expected species projected during Europa flybys, the PNC should approximate best-case $(\mathrm{Ca}=120)$ conditions, which will increase the QITMS partial pressure, and thus MARINE sensitivity, by a factor of four. The PNC field-of-view exceeds fifteen degrees enabling measurements when the spacecraft is pointed off-axis.

The PNC design was also optimized to minimize the effect of hypervelocity (HV) collisions on candidate PNC inner surface materials for the species of relevance in the Europan exosphere and possible plume constituents (e.g. $\mathrm{H}_{2} \mathrm{O}$ ice, $\mathrm{CO}_{2}, \mathrm{SO}_{2}, \mathrm{O}_{2}$ and clathrates of these species). During $\mathrm{HV}$ encounters molecular or ionizing fragmentation due to impact with surfaces can alter the compositional signature of the original gas species, leading to inaccurate parent species assignments. MARINE's inlet system will optimally preserve the parent chemical species, even when entrained in ice grains, unlike the chemical processes experienced in the Cassini INMS experiments whose MS inlet had near normal incidences on titanium-oxide surfaces. For MARINE, a theoretical approach was chosen since it was not feasible to accelerate ice particles and clathrates to the speeds required for such experiments on earth. First-principles quantum mechanical-based reactive dynamics simulation methods have been developed and extensively validated by personnel at the California Institute of Technology. These methods are: ReaxFF, a reactive force field method for simulating general chemical reaction processes [8]; and eFF, the electron force field method for simulating electronically excited states from a mixed quantum-classical non-adiabatic solution to the timedependent Schrodinger's equation. ReaxFF enables the simulation of reactivity, diffusion, material decohesion and fragmentation, and phase transitions, that are essential to capturing the gas or surface chemistry and transport of molecular species during $\mathrm{HV}$ impact events across the spectrometer surfaces. eFF enables an accurate description of large-scale electronically excited processes, such as those expected from ionizing fragmentation during high-energy impacts. Simulations of multiple $\mathrm{H}_{2} \mathrm{O}, \mathrm{CO}_{2}$, and $\mathrm{SO}_{2}$ impacts established that a $5 \mu \mathrm{m}$ Au coated funnel ensured that impacts of single molecules and small (31-100 molecule) water clathrates at velocities less than $6 \mathrm{~km} / \mathrm{sec}$ were specular or quasi-specular. Au also has the least reactivity compared to 


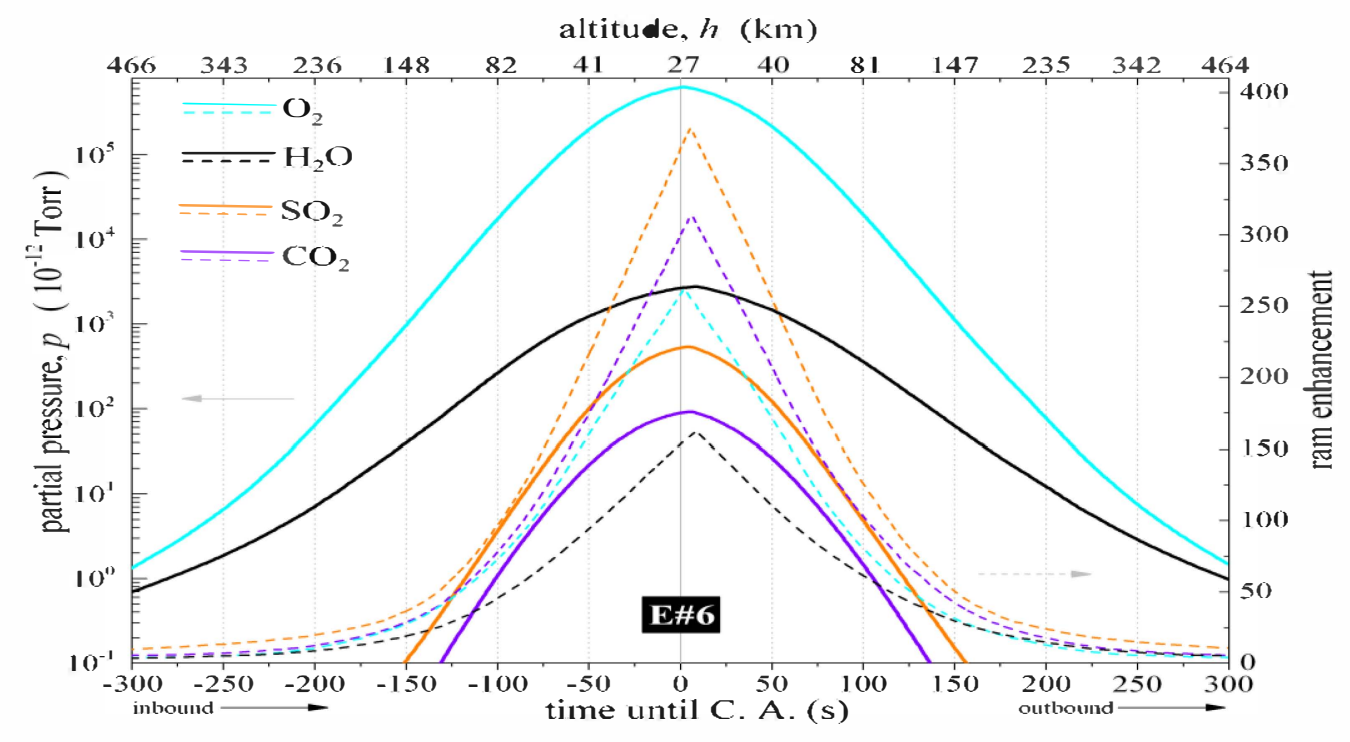

Figure 5. Ram Enhanced Species Density in Funnel (solid curves) and Resultant QITMS Pressure vs. Altitude (dotted curves) for a $25 \mathrm{~km}$ Closest Approach Europa Encounter.

other materials (e.g. Ag, Pt) at impact angles up to $20^{\circ}$ from normal incidence.

JPL's miniaturized QITMS has been under continuous development at JPL's Planetary Surface Instruments (PSI) Group for the last decade. The MARINE instrument inherits its mass spectrometer design from the Vehicle Cabin Atmosphere Monitor (VCAM), an autonomous gas chromatograph mass spectrometer operated continuously aboard the ISS for monitoring the cabin atmosphere for major constituents and trace species from 2010 to 2012 (see Figure 6) [9]. The MARINE electronics are

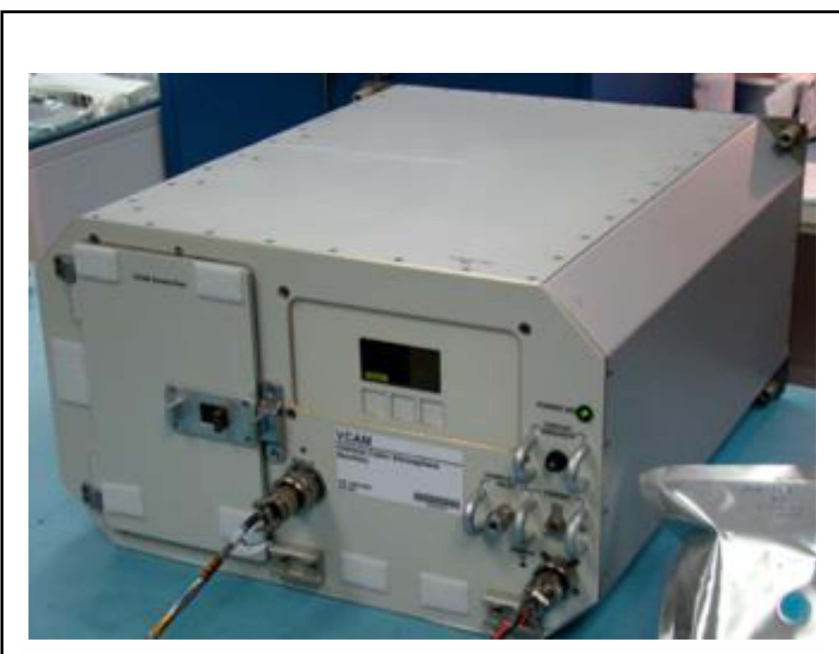

Figure 6. The VCAM Instrument Prior to Flight. VCAM operated continuously aboard the International Space Station (ISS) from 2010 to 2012, performing measurements of the cabin atmosphere's major and trace organic constituents [9]. MARINE's QITMS has been developed from the MS in VCAM. a radiation tolerant design derived from the VCAM electronics, upgraded for high resolution and sensitivity measurements.

The MARINE sensitivity of $1 \mathrm{e} 15$ counts/Torr/sec and dynamic range of $1 \mathrm{e} 6$ is derived from the MS inner volume being effectively a small volume pressure cell (16 $\mathrm{cm}^{3}$ ) maximizing the target density within the trap. The quadrupole ion trap mass spectrometer (QITMS) is constructed entirely of titanium and alumina together with small quantities of non-magnetic stainless steel fasteners. All surfaces that contact analytes are silico-coated to minimize chemical and physical processes. The QITMS uses redundant low power $(<2 \mathrm{~W})$ Ta-disc filament electron sources. The entire QITMS assembly is completely free of any organics and it is routinely baked out to $250^{\circ} \mathrm{C}$ for contamination reduction. The QITMS electron gun delivers $100 \mathrm{uA}$ electron beam tightly focused in the center of the Paul iontrap, that is comprised of three hyperbolic electrodes (ring and two end-cap) mutually insulated and assembled as a pressure cell. Within the trapping volume all created ions are stored by the means of three $r f$ potentials with precisely defined frequencies, amplitudes, and mutual phase differences. The stored ions are ejected from the trap in a mass-dependent fashion by changing the amplitudes of all three $r f$ drives. The ejected ions are detected by MCP operated in a single-count mode. The QITMS was tuned to perform 50 independent trapping cycles per second.

For MARINE, a dual $r f$ frequency architecture has been implemented, where baseline operations has the $r f$ frequency switching between the $650 \mathrm{kHz}$ and $1.2 \mathrm{MHz}$ frequencies every other second, acquiring both WR and WR mass spectra, respectively. The QITMS has highly flexible operational modes with mass range, resolution, sensitivity, repetition rate all programmable and, if desired, can be 
tailored for any encounter via a $10 \mathrm{kbit}$ script file uploaded to the MSEA before the encounter.

MARINE employs a single Photonis Inc. 5901 Spiraltron channel electron multiplier (CEM) for detecting the ions ejected from the QITMS. The CEM is shielded by a multilayer $\mathrm{Al} / \mathrm{Ta} / \mathrm{Al}$ shield to reduce the background radiation-induced count rate to approximately 5 counts/Dalton/sec. The DA design is based on Galileo measurements [10], studies of radiation shielding for ion detectors [11], and radiation modeling for the Europa mission. Radiation testing is planned to validate the detector shielding design. The QITMS provides a high signal to noise ratio (SNR), critical for detecting species at very low abundances. The QITMS acquires a wide range mass spectrum from 20 to 320 Da over 16384 channels, with any mass line having a width of approximately 13 channels. As such, radiation-induced counts will be distributed randomly across these 16384 channels. Based on GEANT4 radiation modeling of the MARINE detector and its shielding design, the radiation induced background rate will be $7 \mathrm{kHz}$, or 5 counts/mass line/sec.

The MARINE MSEA comprises three 6U boards: the Mass Spectrometer Control Electronics (MSCE), the High Voltage Power Board (HVPB), and the Power Distribution Board (PDU). Under 2013 ICEE funding, PT versions of the MSCE and HVPB have been built (see Fig. 4) and successfully validated with the PT QITMS. A breadboard version of the low-risk high-heritage PDU that generates the support voltages (e.g. $\pm 7 \mathrm{~V})$, has been built with testing being deferred to pre-Phase A timeframe in order to concentrate efforts on the more critical MSCE and HVPB assemblies. The MSCE controls the QITMS operational sequences, acquires and handles all instrument data, and communicates with the $\mathrm{S} / \mathrm{C}$. It provides operating voltages to the QITMS and controls the pre-amplifier discriminator electronics. The MSCE provides closed-loop, proportionalintegral-derivative (PID) control of the output $r f$ frequency and amplitude, as well as automatic gain control for the QITMS. The HVPB receives digital control via the MSCE to generate set points for various DC and pulsed voltages and trigger pulse firing for the electrode pulsing. The PT electronics have been developed using JPL best flight practices and incorporated as many components and assemblies as possible from approved planetary mission parts lists. The MSEA radiation shield design uses localized spot shielding of selected electronics parts, to meet the spacecraft vault Total Ionizing Dose requirements.

\section{Performance Testing}

To identify volatiles across a broad mass range, the instrument alternates continuously between two modes that optimize science return. The first mode, shown in Figure 7, offers very high mass resolution $(\mathrm{m} / \Delta \mathrm{m} \approx 4000$ full width half maximum, FWHM) in the low mass region (10-80 Da) where many species of similar mass may exist (e.g. ${ }^{32} \mathrm{~S}$ and ${ }^{16} \mathrm{O}^{16} \mathrm{O}, \mathrm{H}_{2}{ }^{32} \mathrm{~S}$ and ${ }^{34} \mathrm{~S}, \mathrm{H}_{2}{ }^{32} \mathrm{~S}$ and $\left.{ }^{16} \mathrm{O}^{18} \mathrm{O}\right)$. In the high mass resolution mode of operation the ring electrode $r f$ frequency of $1461900 \mathrm{~Hz}$ augmented with the inverted phase-locked end-cap electrode $r f$ potentials at the frequency of $493244 \mathrm{~Hz}$. In the cooling/trapping phase of the $r f$, the cap potentials are approximately 30 volts in amplitude. During the analysis phase in order to maintain a constant optimal resolution over the entire mass range the cap amplitude (nominally $1 \mathrm{~V}$ amplitude) is ramped synchronously with the ring potential.

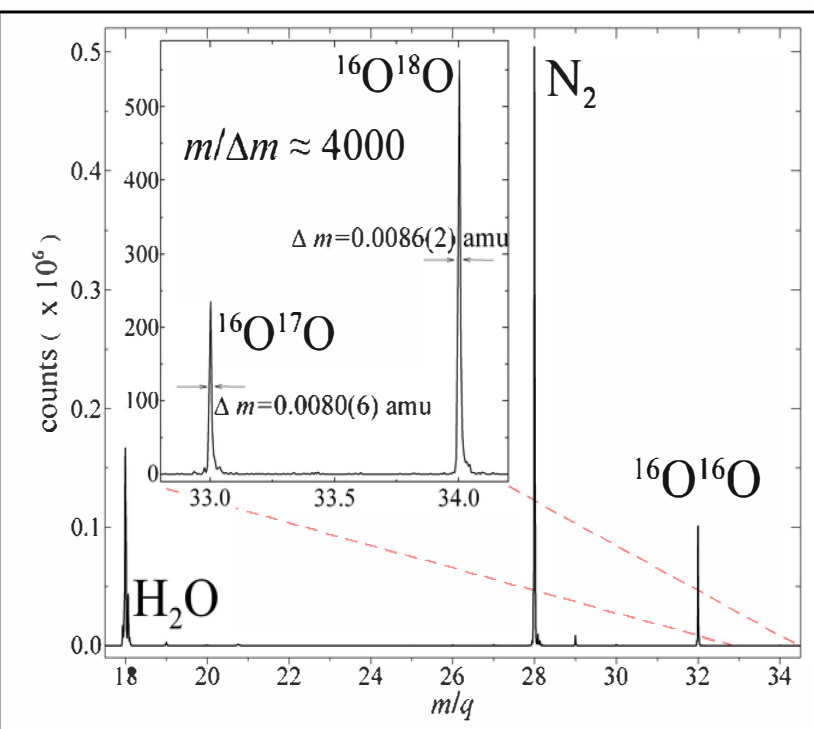

Figure 7. High-Resolution (HR) Mode. This mass spectrum is a portion of one obtained by the MARINE HR mode of operation (10-80 Da). Measurements were made under Europa-relevant densities specifically designed to validate MARINE's ability to meet a requirement to resolve atomic ${ }^{32} \mathrm{~S}$ from $\mathrm{O}_{2}$ at masses 32,33 , and $34 \mathrm{Da}(\mathrm{m} / \Delta \mathrm{m}=2000$ FWHM). The measured MARINE mass resolution $\mathrm{m} / \Delta \mathrm{m}=4000 \mathrm{FWHM}$ exceeds this requirement by $100 \%$.

The second mode, shown in Figure 8, scans a wide mass range $(20-320 \mathrm{Da})$ with a mass resolution $\mathrm{m} / \Delta \mathrm{m} \approx 750$ FWHM. For the wide-range mode of operation with the PFK test gas the ring electrode was driven by $752243 \mathrm{~Hz} r f$ potential whereas the end-cap electrodes were supplied with $253805 \mathrm{~Hz}$ rf potential in phase-inverted mode. In the cooling/trapping phase of the $r f$, the cap potentials are approximately 50 volts in amplitude. During the analysis phase in order to maintain a constant optimal resolution over the entire mass range the cap amplitude (nominally $3 \mathrm{~V}$ amplitude) is ramped synchronously with the ring potential. The measured mass spectrum (solid black curve) is compared with the calculated curve (solid red line) constructed from NIST electron impact ionization dataset folded with the instrumental function. The comparison shows an excellent agreement indicating no signal sensitivity loss at high-mass ranges, which is inherent for this type of mass spectrometer.

In both wide-range and high-resolution modes, no conventionally employed cooling or buffer gas (typically helium) was used. Since the MARINE instrument is designed to operate in the regimes where the ion-ion and ion-neutral 
collisions are negligible, the use of this type of gas is obviated. The measurements using QITMS were performed within ultra-high vacuum (UHV) chamber with base pressure below 1E-11 Torr. The test gas was introduced directly into the trapping volume as in MARINE. The high mass resolution of QITMS stems from the tight focusing of the electron beam in combination with the precise $r f$ waveforms generated for the ring and cap electrodes in both amplitude and relative phase.

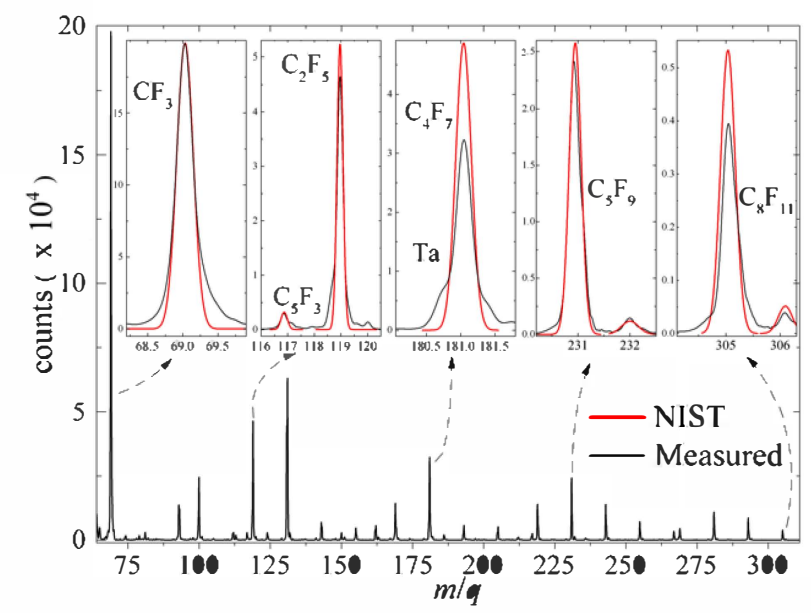

Figure 8. Wide-Range (WR) Mode. This mass spectrum (black curve) demonstrates MARINE operating in the WR mode, measuring perfluorokerosene (PFK). The insets show NIST PFK fragmental relative abundances (convolved with the QITMS lineshape) compared with the measured QITMS mass spectra [14]. Note: QITMS monitoring of Ta emitted by the ionizer filament (third inset) enables MARINE to calibrate its performance during flight. The demonstrated mass resolution of $\mathrm{m} / \Delta \mathbf{m}=$ 750 at $305 \mathrm{Da}$ exceeds the required instrument performance of unit mass resolution with $150 \%$ margin.

\section{Modeled Performance at Europa}

In order to derive the Limits of Detection (LOD) and a performance model for MARINE during flyby encounters of Europa, a system of first order partial differential equations (with respect to time) were solved to derive the number of molecules $\mathrm{N}_{\mathrm{t}}$ of gas species "g" contributing to the partial pressure $p_{\mathrm{t}}$ inside the trap " $\mathrm{t}$ " until the closest approach (CA). The directionality of the molecule velocity $v_{\mathrm{g}(\tau)}$ sampled from the Europan atmosphere is determined by the spacecraft velocity orientation during the flyby, the bulk velocity of particular species (see "model F" of Ref. [12] and Fig. 1 of Ref. [13]), and by thermal velocity component due to temperature variation with altitude. For elucidation purposes, these differential equations can be approximated, reducing them to their algebraic analog in the quasi-static regime, defined as instant of time $\tau_{*}$ at which all first derivatives vanish and the partial pressure of exospheric species "g" inside the trap becomes

$$
\begin{aligned}
& p_{\mathrm{t} *}[\text { Torr }] \approx 16694 \times n_{\mathrm{g}\left(\tau_{*}\right)} k_{B} \sqrt{T_{\mathrm{t}} T_{\mathrm{g}}} \times \\
& C_{\mathrm{a}}\left(0, v_{\mathrm{CA}} / \bar{v}_{\mathrm{g}\left(\tau_{*}\right)}\right) \times v_{\mathrm{CA}} / \bar{v}_{\mathrm{g}\left(\tau_{*}\right)} .
\end{aligned}
$$

In deriving the Eq. (1) we assume that the relative velocity of gas molecules $v_{\mathrm{g}\left(\tau_{*}\right)}$ is equal to the spacecraft velocity $v_{\mathrm{CA}}$ at the closest approach. For example, in the case of the planned Europa-3 Europa encounter and for molecules (like $\mathrm{O}_{2}$ ) whose radial mean velocity is close to zero, the quasi-static regime is reached at the nominal $101 \mathrm{~km}$ (closest approach $\tau_{*} \approx 0$ ). Spacecraft velocity at this instant is $v_{\mathrm{CA}} \approx$ $4.42 \mathrm{~km} / \mathrm{s}$, the thermal velocity of $\mathrm{O}_{2}$ molecules at $T_{\mathrm{g}} \approx 565$ $\mathrm{K}$ is $\bar{v}_{\mathrm{g}(0)} \approx 0.542 \mathrm{~km} / \mathrm{s}$, their number density $n_{\mathrm{g}(0)} \approx 4 \times$ $10^{6} \mathrm{~cm}^{-3}$, and the funnel concentration factor is $C_{\mathrm{a}} \approx 11.5$. Thus according to the approximation Eq. (1), the partial pressure of $\mathrm{O}_{2}$ molecules inside the trap at the temperature $T_{\mathrm{t}}=300 \mathrm{~K}$ is $3.6 \times 10^{-8}$ Torr. Note: this is $28 \%$ higher value that the value returned by the full solution to the partial differential equations due to the approximation. Depending on the thermal velocity of exospheric gas molecules, the enhancement factor $C_{\mathrm{a}}\left(0, v_{\mathrm{CA}} / \bar{v}_{\mathrm{g}\left(\tau_{*}\right)}\right)$ found in Eq. (1) can take values from 1.3 to 14.8 for light and heavy molecules, respectively.

For example, at instant $\tau_{*} \approx 13 \mathrm{~s}$, one finds that this factor peaks at 8.4 for water molecules whose thermal velocity $\bar{v}_{\mathrm{g}\left(\tau_{*}\right)} \approx 0.8 \mathrm{~km} / \mathrm{s}$ corresponds to the temperature of $T_{\mathrm{g}} \approx 694 \mathrm{~K}$. The number density of exospheric water molecules, $n_{\mathrm{g}\left(\tau_{*}\right)} \approx 13.1 \times 10^{4} \mathrm{~cm}^{-3}$, will according to Eq. (1) contribute the trap water partial pressure of $6.4 \times 10^{-10}$ Torr. Note: this result is $26 \%$ higher than the value returned by the full solution where $p_{H 2 O}=5.1 \times 10^{-10}$ Torr. The MARINE performance model for the expected funnel enrichment, resultant QITMS partial pressure and signal rates for a $100 \mathrm{~km}$ CA Europa encounter is shown in Table 1.

For science investigations these limits of detection must also include both background from radiation induced counts on the CEM and the species off-gassed from the spacecraft itself. As has been extensively reported with the Rosetta mission [15] off-gassing forms a background that defines the lower bound limits for certain species LOD (e.g. $\mathrm{H}_{2} \mathrm{O}$ ). The MARINE performance model's predicted limits of detection, including both modeled radiation induced background (see Section 2) and a variety of spacecraft offgassing backgrounds are shown in Table 2 .

Investigations that attempt to discern the presence of localized phenomenon in the Europan exosphere (e.g. potential plumes) or species that might be correlated to surface features (e.g. fracture zones) must be able to detect changes to these species abundances above naturally occurring exospheric abundances. Shown in Table 2 Column D are predicted MARINE 3:1 signal-to-noise $(\mathrm{S} / \mathrm{N})$ LODs above predicted Europan exospheric densities [12,13] for an integrated $6 \mathrm{sec}$ segment of a $27 \mathrm{~km}$ closest approach encounter ( equivalent to $26 \mathrm{~km}$ ground-track). This includes backgrounds due to radiation, $\mathrm{S} / \mathrm{C}$ off gassing, and the 
Table 1. MARINE's Performance Model Signal Rates. ** Published exospheric abundances $[12,13] *$ ) no literaturebased model available and values chosen to illustrate capabilities, a) exospheric density chosen to yield 3:1 source-tobackground offgassing; b) taken as $10 \mathrm{ppm}$ of $\mathrm{H}_{2} \mathrm{O}$; c) $\mathrm{C}_{3} \mathrm{H}_{8}$ taken as representative; d) $\mathrm{C}_{19} \mathrm{H}_{40}$ taken as representative; (e) performance model uses a ram enhancement based on worst-case atomic oxygen-like collisions on the PNC funnel surface. LODs at $10 \%$ measurement error are calculated by requiring a factor of 3 times more ram enhanced pressure than Rosetta-like background, with at least 100 net counts per second signal. A full analytical expression for relating exosphere density to QITMS pressure is given by solving the set of first-order partial derivatives. cps = counts per second.

\begin{tabular}{|c|c|c|c|c|c|}
\hline Species & $\begin{array}{c}\text { Exospheric Density } \\
\text { at } 101 \mathrm{~km} \\
\left(\mathrm{~mol}^{-3} \mathrm{~cm}^{-3}\right)\end{array}$ & $\begin{array}{l}\text { Ram Enhanced } \\
\text { PNC Density }(\mathrm{e}) \\
\left(\mathrm{mol}^{\mathrm{cm}} \mathrm{cm}^{-3}\right)\end{array}$ & $\begin{array}{l}\text { Ram Enhanced } \\
\text { Pressure in QITMS } \\
\text { (Torr) }\end{array}$ & $\begin{array}{l}\text { Rosetta-like Background } \\
\text { Pressure from } \\
\text { Off-gassing } \\
\text { (Torr) }\end{array}$ & $\begin{array}{l}\text { Net } \\
\text { Signal } \\
\text { (cps) }\end{array}$ \\
\hline $\mathrm{H}_{2} \mathrm{O}$ & $1.3 \mathrm{e} 5^{\star *}$ & $1.6 \mathrm{e} 7$ & $5.1 \mathrm{e}-10$ & $2.3 \mathrm{e}-12$ & 253739 \\
\hline $\mathrm{CO}_{2}$ & $175^{* *}$ & $4.9 \mathrm{e} 4$ & $1.5 \mathrm{e}-12$ & $1.9 \mathrm{e}-13$ & 763 \\
\hline $\mathrm{SO}_{2}$ & $500^{* *}$ & $1.8 \mathrm{e} 5$ & $5.7 e-12$ & $1.5 \mathrm{e}-16$ & 2862 \\
\hline $\mathrm{NH}_{3}$ & $351\left(a^{*}\right)$ & 54405 & $1.3 e-12$ & 4.1e-13 & 644 \\
\hline $\mathrm{H}_{2} \mathrm{~S}$ & $1.3\left(b^{*}\right)$ & 293 & $9.2 \mathrm{e}-15$ & $2.2 \mathrm{e}-16$ & 5 \\
\hline$C_{N}(N \leq 6)$ organics & $6\left(c^{*}\right)$ & 1600 & $5.1 e-14$ & $1.7 \mathrm{e}-14$ & 26 \\
\hline$C_{N}(N>6)$ organics & $1.3\left(\mathrm{~d}^{*}\right)$ & 845 & $6.2 \mathrm{e}-14$ & none & 31 \\
\hline
\end{tabular}

Table 2. Performance Model MARINE LODs under Various Conditions. Column A) Includes only Europa radiation induced background at twice the predicted levels $(10$ counts/sec/Dalton) but no $\mathrm{S} / \mathrm{C}$ offgassing. Column B) Includes the radiation induced background in Column $A$ and a $\mathrm{S} / \mathrm{C}$ off-gassing background of $5 \mathrm{e}-14$ Torr for all listed species. Column $C$ ) Includes radiation induced background and $S / C$ offgassing equivalent to that experienced by the ESA Rosetta mission. Column D) Includes all conditions in (C) and the current model density of the target species in the Europa exosphere. Since $\mathrm{H}_{2} \mathrm{O}, \mathrm{SO}_{2}$, and $\mathrm{CO}_{2}$ are major species, the requirement for a LOD of 3:1 signal: background is dominated by their densities at $25 \mathrm{~km}$ altitude, which current models predict as approximately $1 \mathrm{e}^{-3} \mathrm{~cm}^{-3}, 1 \mathrm{e} 5 \mathrm{~cm}^{-3}$ and $1 \mathrm{e} 4 \mathrm{~cm}^{-3}$, for $\mathrm{H}_{2} \mathrm{O}, \mathrm{SO}_{2}$ and $\mathrm{CO}_{2}$, respectively. Column D assumes a 6 sec integrated measurement taking place when the $\mathrm{S} / \mathrm{C}$ is at a closest approach altitude $=27 \mathrm{~km}$. It is possible that when mass spectra integrated across segments of the $\mathrm{S} / \mathrm{C}$ trajectory are examined they may identify the appearance of new species or distinct changes in the abundance profile. This may offer the ability to correlate observed exospheric to surface features identified by other $\mathrm{S} / \mathrm{C}$ instruments (e.g. thermal imager). *A measurement error of $(\mathrm{N} / 100) \%$ assumes an accumulation of at least $(1 / \mathrm{N})^{2}$ net signal.

\begin{tabular}{|c|c|c|c|c|}
\hline & $\mathrm{A}$ & B & C & D \\
\hline Species & $\begin{array}{c}\text { Per second 3:1 S/N LOD } \\
\text { w/ Radiation Background } \\
\text { w/o SIC Offgassing } \\
\text { measurement error } \leq 30 \% \\
\left(\mathrm{~mol}_{\mathrm{cm}} \mathrm{cm}^{-3}\right) \\
\end{array}$ & $\begin{array}{l}\text { Per second 3:1 SIN LOD } \\
\text { w/ Radiation Background } \\
\text { w 5e-14 Torr Offgassing } \\
\left.\text { (mol. } \mathrm{cm}^{-3}\right) \\
\end{array}$ & $\begin{array}{l}\text { Per second 3:1 S/N LOD } \\
\text { W/ Radiation Background } \\
\text { w Rosetta Offgassing [15] } \\
\quad\left(\mathrm{mol}_{\mathrm{cm}} \mathrm{cm}^{-3}\right) \\
\end{array}$ & $\begin{array}{c}6 \text { Second Integrated 3:1 LOD } \\
\text { against Europa Exosphere } \\
\text { w/ Radiation Background } \\
\text { w Rosetta Offgassing } \\
\left(\mathrm{mol}^{\mathrm{cm}} \mathrm{cm}^{-3}\right)\end{array}$ \\
\hline $\mathrm{H}_{2} \mathrm{O}$ & 5.6 & 38 & 1759 & 2100 \\
\hline $\mathrm{CO}_{2}$ & 2.5 & 18 & 67 & 300 \\
\hline $\mathrm{SO}_{2}$ & 1.9 & 13 & 5 & 900 \\
\hline $\mathrm{NH}_{3}$ & 6 & 41 & 332 & 55 \\
\hline $\mathrm{H}_{2} \mathrm{~S}$ & 3.1 & 21 & 3 & 1 \\
\hline $\begin{array}{c}\mathrm{C}_{\mathrm{N}} \\
(\mathrm{N} \leq 6) \\
\text { organics }\end{array}$ & 2.5 & 18 & 3 & 1 \\
\hline $\begin{array}{c}C_{N} \\
(N>6) \\
\text { organics }\end{array}$ & 0.5 & 3 & 0.5 & 0.2 \\
\hline
\end{tabular}


predicted Europan exosphere. Due to high sensitivity and ram enhanced exospheric sampling it is predicted that MARINE can detect 3\% and 1\% changes in Europan $\mathrm{CO}_{2}$ and $\mathrm{SO}_{2}$ when mass spectra are integrated over segments of the CA ground track. This is critical, as it may be possible to use these integrated spectra to identify the appearance of new species or distinct changes in the abundance profiles which correlate to surface features identified by other $\mathrm{S} / \mathrm{C}$ instruments (e.g. radar, spectroscopic, or thermal imagers). In the model, we assume the published Europan abundance profiles. It is planned that during the actual mission, these

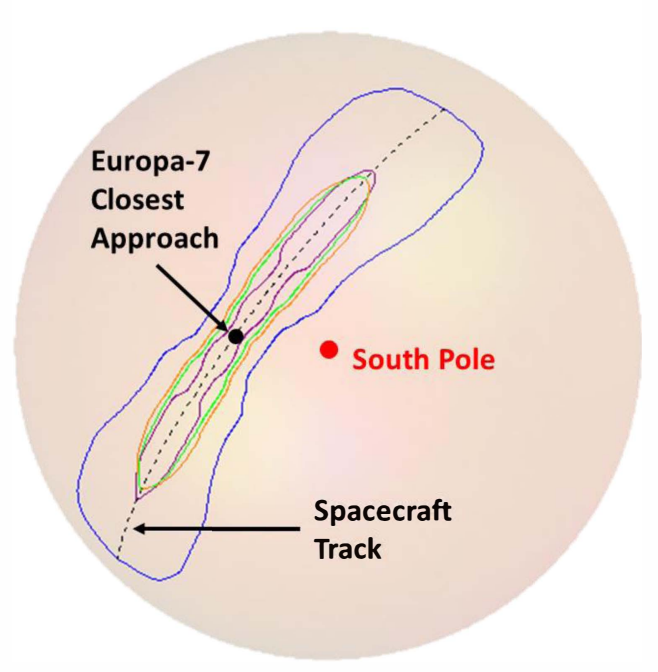

Figure 9. Modeled MARINE Plume Detection. During the flyby (dotted black line) if a plume existed anywhere ON the blue ellipse, then MARINE will detect plume $\mathrm{H}_{2} \mathrm{O}$ as peak in the exospheric $\mathrm{H}_{2} \mathrm{O}$ abundance vs. altitude profile with $3: 1 \mathrm{~S} / \mathrm{N}$ and at most $10 \%$ statistical error. If a plume existed anywhere INSIDE the blue ellipse, then MARINE would detect plume $\mathrm{H}_{2} \mathrm{O}$ at greater than $3: 1 \mathrm{~S} / \mathrm{N}$. The blue ellipse is approximately $2850 \mathrm{~km} \times 285 \mathrm{~km}$ in dimension.

MARINE will detect plume-based $\mathrm{NH}_{3}$ and most $\mathrm{C}_{\mathrm{N}}$ $(\mathrm{N} \leq 6)$ organics at concentrations $1 \mathrm{e}-5$ with respect to (w.r.t) $\mathrm{H}_{2} \mathrm{O}$ (or greater) if a plume existed within the region bounded by purple ellipse $(1800 \mathrm{~km} \times 80 \mathrm{~km})$. MARINE will detect plume-based $\mathrm{CO}_{2}$ at concentrations 1e-5 w.r.t $\mathrm{H}_{2} \mathrm{O}$ (or greater) if a plume existed within the region bounded by green ellipse $(1670 \mathrm{~km} \times 140 \mathrm{~km})$. MARINE will detect plumebased $\mathrm{SO}_{2}$ and most $\mathrm{C}_{\mathrm{N}}(\mathrm{N}>6)$ organics at concentrations 1e-5 w.r.t $\mathrm{H}_{2} \mathrm{O}$ (or greater) if a plume existed within the region bounded by yellow ellipse $(1600 \mathrm{~km} \times 170 \mathrm{~km})$. The smaller green and purple ellipses are predominantly due to the inclusion of Rosetta-like S/C offgassing in the performance model, where species like $\mathrm{NH}_{3}, \mathrm{CO}_{2}$, and $\mathrm{C}_{\mathrm{N}}(\mathrm{N} \leq 6)$ organics have higher background pressures than $\mathrm{SO}_{2}$ and $\mathrm{C}_{\mathrm{N}}$ $(\mathrm{N}>6)$ organics. abundance profiles would be measured by MARINE during the first flyby encounters.

The ability of MARINE to discern these small deviations in the exospheric abundances enables a highly sensitive investigation to detect and characterize any potential plumes. Shown in Figure 9 is a simulated encounter corresponding to the Europa-7 trajectory through the southern hemisphere of Europa as defined in the Europa Proposal Information Package (PIP). If plumes do exist, the MARINE instrument is a powerful tool for detecting their presence.

\section{SUMMARY}

Mass spectrometry is uniquely suited for in situ measurements of planetary bodies with extremely tenuous atmospheres. The scientific exploration of Europa's environment for definitive characterization, and assessment of habitability levies extreme requirements on a mass spectrometer for parameters such as sensitivity and mass $r$ esolution. The MARINE instrument, designed, built, and tested with the support of the 2013 ICEE award, exceeds all of these requirements with margins ranging from 100 to $1000 \%$, while remaining fully compatible with spacecraft accommodation constraints for mass, power, data volume, and field-of-view. The MARINE instrument using optimal ram enhancement of neutrals for high signal-to-S/C offgassing backgrounds and a highly sensitive MS, yields a per-second sensitivity better than 1 molecules $\bullet \mathrm{cm}^{-3} \cdot \mathrm{s}^{-1}$ in the Europan radiation environment. This is at least 100 times greater than other types of MS instruments that might be flown [16]. Through a combination of discovery driven and hypothesis-driven approaches, MARINE will vastly increase our knowledge of Europa's exosphere by providing maps of exospheric elements, isotopes, and molecules.

\section{ACKNOWLEDGEMENTS}

This research was carried out at the Jet Propulsion Laboratory, California Institute of Technology, under a contract with the National Aeronautics and Space Administration.

\section{REFERENCES}

[1] Europa Clipper Science Traceability Matrix, Controlled v.2.2 - June $10, \quad 2014$. (https://solarsystem.nasa.gov/europa/sdt2013.cfm/)

[2] Committee on the Planetary Science Decadal Survey; National Research Council (2011). Vision and Voyages for Planetary Science in the Decade 2013-2022. (http://www.nap.edu/openbook.php?record_id=13117)

[3] Roth, L., Saur, J., Retherford, K.D., Strobel, D.F., Feldman, P.D., McGrath, M.A. \& Nimmo, F. (2014). Transient water vapor at Europa's south pole. Science $343,171-174$.

[4] Waite, J.H. Jr. et al. (2006). Cassini Ion and Neutral Mass 
Spectrometer: Enceladus plume composition and structure. Science 311, 1419-1422.

[5] Johnson, R.E., et al., (2009). Composition and Detection of Europa's Sputter-Induced Atmosphere. In: Poppalardo, R., et al. (Eds.), Europa, University of Arizona Press, Tucson.

[6] Dalton III, J.B., Shirley, J.H. \& Kamp, L.W. (2012). Europa's icy bright plains and dark linea: Exogenic and endogenic contributions to composition and surface properties. Journal of Geophysical Research 117, E03003.

[7] Pilinski, M.D. \& Argrow, B.M. (2013). Aerodynamic analysis based on challenging minisatellite payload satellite lift-to-drag measurements. Journal of Spacecraft and Rockets 50, 1162-1170.

[8] van Duin, W., et al. (2001). ReaxFF: A reactive force field for hydrocarbons. J. Phys Chem A 105, 93969409.

[9] Darrach MR., et al (2012) Trace Chemical and Major Constituent Measurements of the International Space Station Atmosphere by the Vehicle Cabin Atmosphere Monitor. J. Am. Inst. Aeronautics and Astronautics. Electronic 2012-3432.

[10] Boldt, J., et al. (2008). Assessment of Radiation Effects on Science and Engineering Detectors for JEO, Jet Propulsion Laboratory.

[11] Benke, R.R., et al. (2013). Comparison of Radiation Induced Noise Levels in Two Ion Detectors for Shielded Space Instruments in High Radiation Fields. IEEE Transactions on Nuclear Science 60, 365

[12] Shematovich, V.I., Johnson, R.E., Cooper, J.F. \& Wong, M.C. (2005). Surface-bounded atmosphere of Europa. Icarus $173,480-498$.

[13] Cassidy, T.A., Johnson, R.E., McGrath, M.A., Wong, M.C. \& Cooper, J.F. (2007). The spatial morphology of Europa's near-surface O2 atmosphere. Icarus 191, 755764.

[14] Madzunkov, S.M. \& Nikolić, D. (2014). Accurate Xe isotope measurement using the JPL ion trap. Journal of The American Society for Mass Spectrometry, in press. DOI: 10.1007/s13361-014-0980-2.

[15] Schlappi, B., et al. (2010). Influence of spacecraft outgassing on the exploration of tenuous atmospheres with in situ mass spectrometry. Journal of Geophysical Research: Space Physics A12, 115.

[16] Wurz, P., et al. (2014). "Measurement of the Atmospheres of Europa, Ganymede, and Callisto", in European Planetary Science Congress, Cascais, Portugal.

\section{BIOGRAPHY}

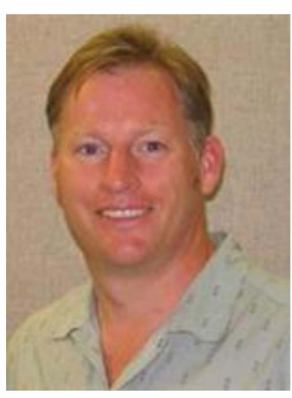

Murray Darrach received his PhD in Physics from the University of Windsor (Canada) in 1990 and joined JPL in 1993. He is JPL Principal Technologist and leads the JPL Planetary Surface Instruments Group, a collection of 12 scientists and engineers developing a variety of miniature sensors for planetary exploration. Dr. Darrach has delivered two JPL flight mass spectrometers, the Trace Gas Analyzer in 2000 and the Vehicle Cabin Air Monitor in 2009. He is the Principal Investigator (PI) for the VCAM follow-on, the micro Total Atmosphere Monitor (microTAM), which will be launched to the International Space Station in 2018 and the Deputy PI for the MARINE investigation.

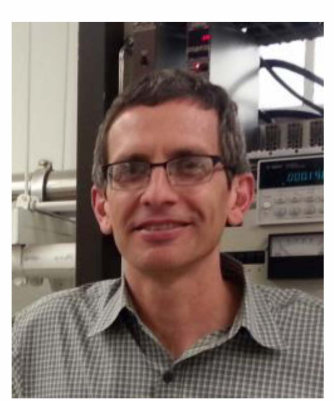

Ken Farley is the W.M. Keck Foundation Professor of Geochemistry in the Division of Geological and Planetary Sciences at the California Institute of Technology. His research centers on development and application of geochemistry techniques, especially involving isotopes of the noble gases, to a wide range of terrestrial and solar system questions. Specific areas of interest include geochronology of both Earth and Mars, the geochemical evolution of the Earth, and the behavior of noble gases in minerals. He is currently a participating scientist on the Mars Science Laboratory mission and is project scientist for the Mars 2020 Science Rover mission. He is the PI for the MARINE investigation.

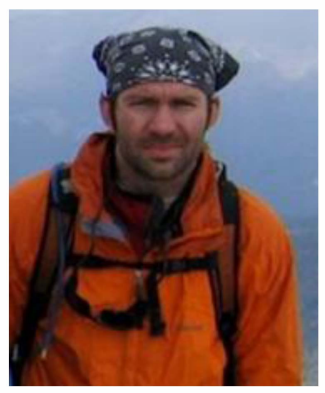

Rembrandt Schaefer received $h s$ B.S. in Computer and Systems Engineering from Rensselaer Polytechnic Institute in 1999. In 2004 he joined JPL where he is currently a member of the Advanced Computer Systems \& Technologies group at JPL. His specializations include mixed signal electronics and system design for scientific instrumentation and flight electronics. Mr. Schaefer's present work includes high-speed analog front-end design. He is the principal architect for the MARINE electronics and led a team of six engineers in developing and testing the prototype MARINE electronics. Mr. Schaefer is a CoInvestigator for the MARINE investigation. 


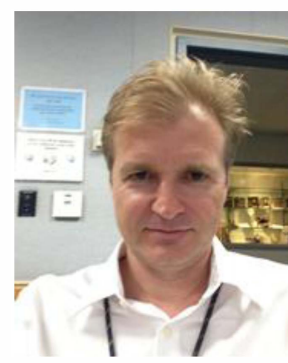

Stojan Madzunkov received his PhD in Physics from the University of Stockholm in 2004. In 2005 he joined JPL where he is currently a Senior Technologist in the Planetary Surface Instruments Group. Dr. Madzunkov was an Instrument Scientist for VCAM and solely responsible for developing the major constituents hardware and analysis capabilities for the instrument. Dr. Madzunkov led the design and testing of the prototype MARINE quadrupole ion trap mass spectrometer. He was the Principal Investigator for the microGas Monitor, a micro-machined gas chromatograph and mass spectrometer for planetary and crewed space exploration. He is the D-PI for microTAM and the Instrument Scientist for the MARINE investigation.

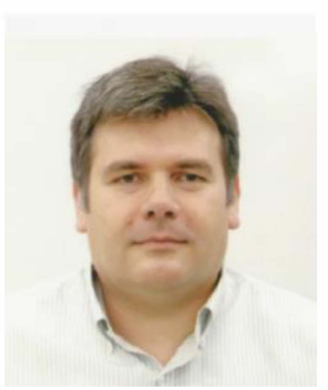

Dragan Nikolić is a researcher at JPL. He received his B.S. in physics at the University of Novi Sad (1994), a M.S. in plasma physics (1998) at the University of Belgrade, and a Ph.D. in relativistic atomic physics (2004) under Prof. E. Lindroth at the Stockholm University. In 2013 he joined JPL to pursue his interests in design, modeling, and operation of miniature highresolution mass spectrometers in space exploration. Dr. Nikolic led the performance modeling effort for MARINE.

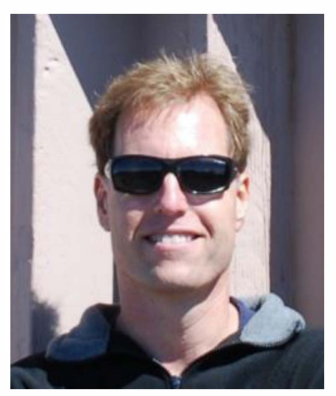

Richard D. Kidd is a research scientist at NASA's Jet Propulsion Laboratory (JPL). He received his Ph.D. in Molecular and Cell Biology at the Pennsylvania State University (1996) where he carried out research in protein crystallography and joined JPL in 2004. Dr. Kidd's background is quite varied ranging from microfluidics, biomedical and biochemical science to instrumentation and spaceflight, with settings (both in the laboratory and in the field) in private industry, university and government. He is interested in the scientific exploration of space, in particular, the research, planning, testing and/or operations of in situ instrumentation. Dr. Kidd was the MARINE proposal manager and member of the systems engineering team.

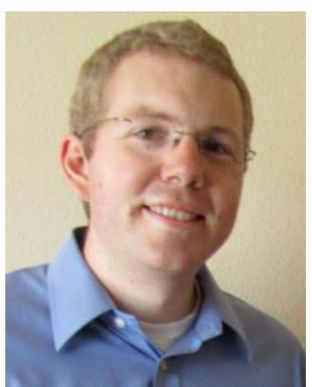

Evan L. Neidholdt is a research technologist at JPL. He received his B.A. in Chemistry and Mathematics from Concordia University Irvine, 2005. He completed Ph.D studies with J.L. Beauchamp at the California Institute of Technology, receiving his Ph.D from Caltech in 2010. At Caltech he developed two novel ambient ionization sources for detection of trace explosives, nerve agents, and other organics in the field using mass spectrometry. He joined the technical staff at JPL following completion of thesis work and continues to pursue his interests in space mass spectrometer instrument and sampling technologies development at JPL. Dr. Neidholdt led the development of the MARINE MSHA prototype and was the MARINE Systems Engineer.

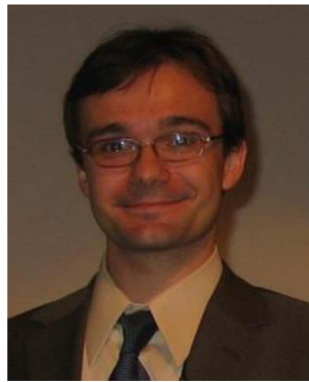

Marcin Pilinski is the Group Leader for the Spacecraft Gas-Surface Interaction and Aerodynamic Modeling Standards working group at ASTRA (Atmospheric \& Space Technology Research Associates, LLC). As part of this effort he is leading an international team of experts in the determination of bestpractices and investigation of possible ISO standards for LEO spacecraft gas-surface interactions and aerodynamic models. He received his Ph.D in Aerospace Engineering Sciences from the University of Colorado in 2011. Using his expertise in hypervelocity spacecraft gassurface interactions, Dr. Pilinksi led the effort to model and design the MARINE PNC collector and inlet system.

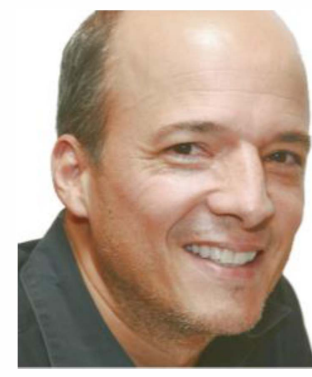

Andres Jaramillo-Botero was born in Cali, Colombia, in 1964. He received a B.S. degree in Electrical Engineering from Boston University in 1986, an M.S. degree in Computer Science as a Fulbright scholar from the State University of New York in 1990, and a Ph.D. degree in Engineering from the Universidad Politecnica de Valencia (Valencia, Spain) in 1998. In 2006, he joined Caltech as a Senior Research Scientist in the Chemistry and Chemical Engineering division and as the Director of Multiscale Science and Simulation at the Materials and Process Simulation Center (MSC). His research interests include non-adiabatic dynamics of molecules and materials, hypervelocity impact dynamics, low-temperature material growth, nanoscale systems characterization and properties optimization from firstprinciples modeling and simulation, and control at the nanoscale. 


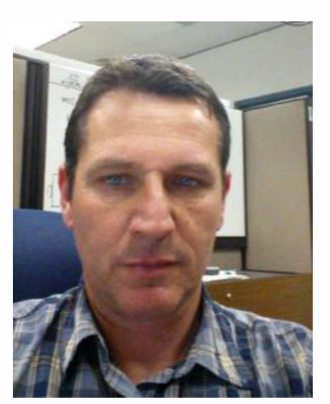

Jurij Simčič is a researcher at JPL. He received his B.S. (1997) in physics, M.S. (2003) in particle beam physics and Ph.D. (2005) in particle beam physics and beam analytical methods from the University of Ljubljana, Slovenia. During his graduate studies he held a research assistant position at the department of low and medium energy physics at J. Stefan Institute in Ljubljana. He became a NASA postdoctoral fellow in Atomic and molecular physics group at JPL in 2006 and an employee in 2008. In 2013 he joined Planetary Surface Instruments group at JPL working on the design of quadrupole ion traps for miniature mass spectrometers in space exploration. Dr. Simčič was member of the MARINE MSHA design team and led the modeling effort for the radiation shielding of the MARINE detectors and electronics. 
